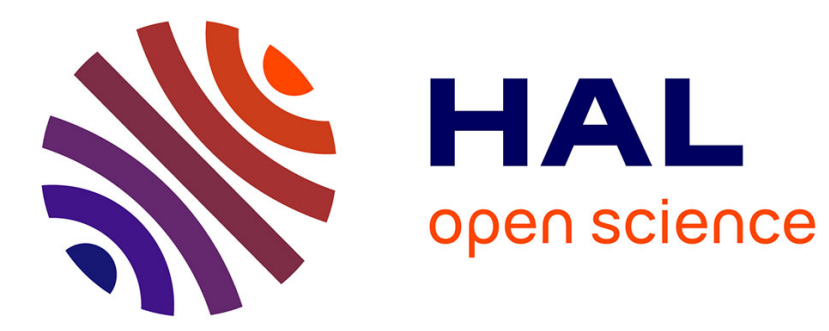

\title{
Reference Airspeed Setting For Time Constrained Descent at Idle Thrust
}

Thierry Miquel

\section{To cite this version:}

Thierry Miquel. Reference Airspeed Setting For Time Constrained Descent at Idle Thrust. AIAA GNC 2013, AIAA Guidance, Navigation and Control Conference, Aug 2013, Boston, United States. pp xxxx. hal-00917976

\section{HAL Id: hal-00917976 \\ https://hal-enac.archives-ouvertes.fr/hal-00917976}

Submitted on 12 Dec 2013

HAL is a multi-disciplinary open access archive for the deposit and dissemination of scientific research documents, whether they are published or not. The documents may come from teaching and research institutions in France or abroad, or from public or private research centers.
L'archive ouverte pluridisciplinaire HAL, est destinée au dépôt et à la diffusion de documents scientifiques de niveau recherche, publiés ou non, émanant des établissements d'enseignement et de recherche français ou étrangers, des laboratoires publics ou privés. 


\title{
Reference Airspeed Setting For Time Constrained Descent at Idle Thrust
}

\author{
Thierry Miquel ${ }^{1}$ \\ Ecole Nationale de l'Aviation Civile, Toulouse, France
}

This paper addresses the issue of computing a reference airspeed for time constrained descent at idle thrust. More specifically, we will assume a futuristic 4D trajectory application where the air traffic controller will ask an aircraft to overfly a meter fix at a specific time; this time is assumed to be specified by the air traffic controller to settle properly the arrival sequence and could be given for example through an Arrival Manager (AMAN). In addition, this clearance is assumed to be given after the Top Of Descent (TOD) of the aircraft. The aircraft is assumed to descent at idle thrust and has to overfly the meter fix at a given altitude and airspeed to be correctly prepared to land. Idle thrust enables to reduce noise, fuel burn and emissions. A BADA model of an Airbus A320 is used to illustrate the proposed design.

\section{Introduction}

$\mathrm{N}_{\mathrm{in}}$ owadays, environmental impact and efficiency have become the two very important aspects in aviation industry after safety. Continuous Descent Operation (CDO) is an optimized procedure that can both bring environmental benefits by reducing noise and engine emission and improve airport efficiency by better using of airspace and arrival route placement. CDO is defined as 'an aircraft operating technique aided by appropriate airspace and procedure design and appropriate ATC clearances enabling the execution of a flight profile optimized to the operating capability of the aircraft, with low engine thrust settings and, where possible, a low drag configuration, thereby reducing fuel burn and emissions during descent. The optimum vertical profile takes the form of a continuously descending path, with a minimum of level flight segments only as needed to decelerate and configure the aircraft or to establish on a landing guidance system (e.g. ILS). ${ }^{[1]}$

As a result of the potential benefits, studies ${ }^{[2]}$ at a number of airports began in approximately 1999, and continued through 2004-2010. By September 2010, CDO procedures have been established at 79 airports around Europe. A further 59 airports are either committed to evaluate feasibility of implementing CDO, or in the process of formalizing the $\mathrm{CDO}$ if it is already being offered. In addition, $\mathrm{CDO}$ is also considered as one of the essential building blocks for the Single European Sky.

European stakeholders have initiated a European CDO implementation program. In September 2008, CANSO, IATA and Eurocontrol signed up a Flight Efficiency Plan that includes a specific target to increase European CDA (Continuous Descent Approach) performance and achievement. This resulted in the publication and launch in 2009 of an European Joint Industry Action Plan, which built on the high-level commitments and set out specific actions for the European Aviation Industry to ensure CDA's rapid deployment. Up to now, Basic CDO is already in operation at UK Heathrow Airport ${ }^{[3]}$. In Sweden the European project NUP2 has enabled SAS and LFV to operate 4D flight paths, or green approaches, at Stockholm-Arlanda airport with beneficial impacts on environment. Thanks to the collaboration between Air France and DSNA, CDAs are operated routinely at Marseille airport in France.

In United States, a program known as Partnership for AIR Transportation Noise and Emission Reduction (PARTNER), also conducted field tests at Louisville International Airport in 2002 and at Los Angeles International Airport in $2007^{[4]}$.

NASA and FAA have been involved in extensive efforts to develop advanced concepts, technologies and procedures for the Next Generation Air Transportation System (NextGen) later on. One aim of NextGen is to develop ground-side automation systems to assist controllers in strategic planning operations. The En Route Descent Advisor (EDA) is one of the Center TRACON Automation System (CTAS) decision support tools under development at the NASA Ames Research Center. EDA generates maneuver advisories for arrival aircraft to meet

\footnotetext{
${ }^{1}$ Ecole Nationale de l'Aviation Civile, MAIAA Lab, thierry.miquel@enac.fr

Copyright $\odot 2013$ by ENAC. Published by the American Institute of Aeronautics and Astronautics, Inc., with permission.
} 
scheduled arrival times at the arrival meter fix, sometimes $20-25$ minutes ahead of the aircraft's scheduled meter fix arrival time ${ }^{[5]}$. A research ${ }^{[6]}$ has also been done to develop ground-side automation to enable 4D-TrajectoryBased Operations (4DTBO) in terminal airspace. This research developed and illustrated a computational framework for the design of 4D-Trajectories (4DTs) based on fundamental flight mechanics and nonlinear trajectory optimization techniques with sample scenarios. Furthermore, the 4D-trajectory design process is based completely on open-source models.

AIRE Project (Atlantic Interoperability Initiative to Reduce Emissions) is a joint initiative by the European Commission and the FAA to improve energy efficiency and aircraft noise. AURORA project was a project implemented by Airbus, Scandinavian Airlines International (SAS), Swedish ANSP LFV and Stockholm Arlanda Airport. They conducted Continuous Descent Approaches for the first time on SAS transatlantic flights using an Airbus A330.

Another new ATC technique called Point merge system (PMS) ${ }^{[7]}$ aimed to facilitate the merging of traffic from a number of Area Navigation (RNAV) arrival routes. The technique is based upon aircraft flying a quasi-arc, up to $30 \mathrm{NM}$ long, with a radius of more than $20 \mathrm{NM}$ from the designated merging point. Each arc has a published altitude that the aircraft must have reached before establishing on the arc and a predefined speed to fly it. In general the arc nearest to the merging point has the highest altitude while the other has the lowest altitude so that the external sequencing leg is free from traffic from the internal sequencing leg during descent of the traffic. On April 7, 2011, Oslo became the world's first airport to implement a PMS in their airspace. Other countries and airports will follow.

The vertical trajectory optimization has also been studied to maximize the benefits coming from CDA procedure. In some research ${ }^{[8]}$, idle power during the descent is maintained while several optimal trajectories for a CDA are presented in order to maximize the benefits in terms of operating cost such as fuel consumption or flight time. To obtain optimal trajectories, problems are formulated as multiple phase optimal control problems with a fixed range and include considerations of both operating conditions and speed constraints. The altitude optimization range is from cruise altitude to the intercept the Instrumental Landing System (ILS) glide slope. By dividing the optimal trajectory into two flight segments, cruise and descent, both the position of the Top Of Descent (TOD) and the subsequent optimal descent path were obtained simultaneously. Methods of trajectory optimization have also been developed by others researchers. In a research considering "4D Green Approach Trajectory" ${ }^{[9]}$, the task of merging an aircraft over a specified meter fix is addressed through a novel ATS clearance in which air traffic control clears an aircraft to track an ad-hoc computed reference trajectory. This enables the aircraft to merge at a specified meter fix at a given time. This envisioned application relies on 4D operations and needs at least two functions to be operated: a function which computes the reference trajectory, which can be located either on the ground within the Arrival MANager (AMAN) on onboard the aircraft within the Flight Management System (FMS), and a function which enables the aircraft to track the reference trajectory. In comparison with current operations, the change is that the controller would communicate decisions on traffic flow organization at a higher level to the pilot rather than requiring the controller to calculate and communicate lower-level guidance instructions. The main benefit expected from this application is to improve flight efficiency by more precise maneuvering resulting from onboard capabilities, and also noise abatement and fuel saving ${ }^{[14]}$. Moreover, moving from radar vectoring to monitoring precomputed trajectories would contribute to decrease controller's workload, and therefore to increase sector capacity.

This paper addresses the issue of computing a reference airspeed for time constrained descent at idle thrust. More specifically, we will assume a futuristic 4D trajectory application where the air traffic controller will ask an aircraft to overfly a meter fix at a specific time; this time is assumed to be specified by the air traffic controller to settle properly the arrival sequence and could be given for example through an Arrival Manager (AMAN). In addition, this clearance is assumed to be given after the Top Of Descent (TOD) of the aircraft.

The aircraft is assumed to descent at idle thrust and has to overfly the meter fix at a given altitude and airspeed to be correctly prepared to land. Idle thrust enables to reduce noise, fuel burn and emissions. This is a two boundaries control problem (initial and final conditions are set), which is known to be quite difficult to solve.

From the Air traffic control side, this kind of application will relieve air traffic controllers of providing time consuming radar vectoring instructions to the aircraft once the flight crew has accepted the $4 \mathrm{D}$ clearance. Thus, the expected benefit of such new capabilities is an increase of air traffic controller availability, which could result in increased air traffic efficiency and / or capacity. 


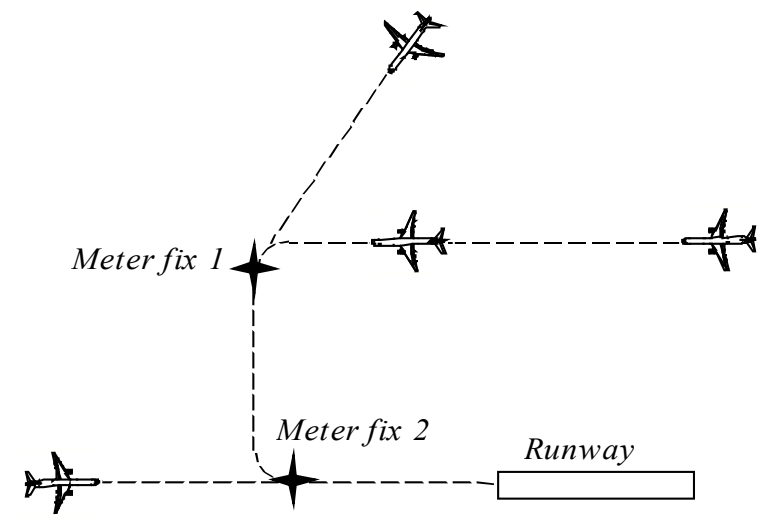

Fig. 1: Sequencing and merging operations for arriving aircraft at an airport

The paper is organized as follows: in the second section, the trajectory control strategy of an aircraft at idle thrust is developed. Then an airspeed control law through commanded flight path angle is developed; basically, a proportional and integral (PI) controller is dedicated to this function. The computation of the reference airspeed to solve the two boundaries control problem is addressed in the fourth section. Illustrative examples are then presented in order to show the effectiveness (and also the weakness) of the proposed approach. The aircraft model which is used as an example is an Airbus A 320 modeled through BADA ${ }^{[10]}$. Finally conclusions are drawn and future work is presented.

\section{Trajectory Control Strategy}

Assuming a constant wind, the second Newton's law of motion applied on an aircraft modeled as a point-mass results in the following expression for the along track acceleration, where $V$ denotes the airspeed, $T$ the thrust, $D$ the drag, $m$ the aircraft, $g$ the acceleration of gravity and $\gamma$ the flight path angle:

$$
\dot{V}=\frac{T-D}{m}-g \sin (\gamma)
$$

Altitude rate is defined as:

$$
\dot{h}=V \sin (\gamma)
$$

If throttle setting is specified, the along track acceleration only depends on the altitude rate:

$$
\dot{V}=\frac{d V}{d h} \dot{h}=\frac{d V}{d h} V \sin (\gamma)
$$

Combining those two equations leads to the well known total energy model used by the aircraft performance data BADA (Base of Aircraft Data $\left.{ }^{[10]}\right)$ :

$$
\frac{d V}{d h} V \sin (\gamma)=\frac{T-D}{m}-g \sin (\gamma) \Leftrightarrow T-D=m\left(g+V \frac{d V}{d h}\right) \sin (\gamma)
$$

Aerodynamic drag force $D$ is given as the product of the drag coefficient, $C_{D}$, and the dynamic pressure as:

$$
D=\frac{1}{2} \rho S V^{2} C_{D}
$$

where $\rho$ is the density of air and $S$ is the wing reference area. The drag coefficient $C_{D}$ is given as the sum of zerolift drag coefficient, $C_{D 0}$, and the induced drag coefficient, which is usually taken as a quadratic function of the lift coefficient, $C_{L}$. Thus:

$$
C_{D}=C_{D 0}+C_{D 2} C_{L}^{2}
$$

Both coefficients $C_{D 0}$ and $C_{D 2}$ are functions of aerodynamic configuration of the aircraft. Traditionally, drag coefficients are given as a function of Mach and Reynolds number. BADA models these values as constants for each of the aerodynamic configurations which are: takeoff, initial climb, clean, approach and landing. BADA provides altitude and speed thresholds for determining these aerodynamic configurations. Lift coefficient $C_{L}$ is obtained using the definition of lift, assuming that lift is equal to weight and small flight path angle: 


$$
C_{L}=2 \frac{L}{\rho S V^{2}}=2 \frac{m g}{\rho S V^{2}}
$$

BADA also specifies maximum thrust during climb as a quadratic function of altitude. The general expression is:

$$
T_{\max }=C_{T 1}\left(1-\frac{h}{C_{T 2}}+C_{T 3} h^{2}\right)
$$

A fraction of the maximum thrust is used for idle-thrust descent. It is given as:

$$
T_{\text {idle }}=C_{T 4} T_{\max }
$$

where $C_{T I}$ through $C_{T 4}$ are aircraft-specific coefficients.

Minimum fuel flow corresponding to idle thrust descent conditions is also provided by BADA. The idle thrust part of the descent stops when the aircraft switches to approach and landing configuration at which point thrust is generally increased. Denoting $h$ the pressure altitude, the minimum fuel flow $f_{\min }(\mathrm{kg} / \mathrm{min})$ for idle-thrust is:

$$
f_{\text {min }}=C_{f 3}\left(1-\frac{h}{C_{f 4}}\right)
$$

The following figure come from the Airbus A320 model provided by BADA ${ }^{[10]}$ : it represents the values of drag and idle thrust (in Newton) as a function of the true airspeed taken between 180 knots and 340 knots and for a mass of 64 tons and two values of the altitude pressure: 10000 and 3000 feet.

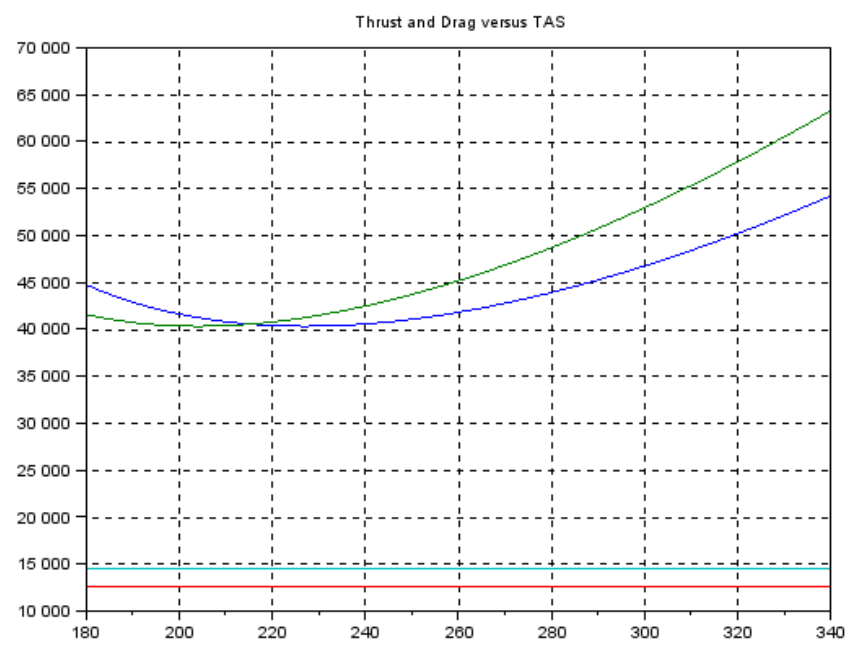

Fig. 2: BADA model for Airbus A 320: thrust and drag as a function of the airspeed

\section{Airspeed Control at Constant Thrust}

On the following figure, thrust $T$ and drag $D$ are plotted as a function of airspeed $V$; obviously, this presents the general shape of Fig. 2. It is worth noticing that equation (4) indicates that the difference $T-D$ is proportional to the sine of the flight path angle $\gamma$; in addition, it is clear that any change in the flight path angle $\gamma$ induces changes in airspeed $V$ as well as in altitude rate (as soon as $\dot{h}=V \sin (\gamma)$ ). As a consequence, flight path angle $\gamma$ can be envisioned as an outer loop control of both airspeed and altitude rate. 


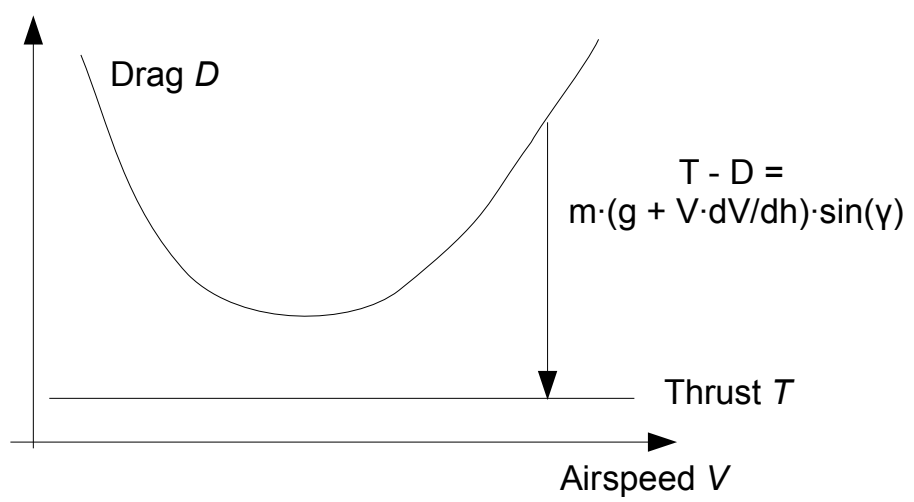

Fig. 3: Thrust and drag as a function of the airspeed

In this section, we present a control loop which enables the aircraft to achieve a commanded airspeed through the control of the flight path angle. Assuming small flight path angle $\gamma$, the longitudinal equation of motion of the aircraft (1) reads as follows, where $T$ is the thrust, $D$ the drag, $m$ the mass of the aircraft, $g$ the acceleration of gravity and $\gamma$ the flight path angle:

$$
\dot{V} \approx \frac{T-D}{m}-g \gamma
$$

To design the speed control law using linear control techniques, we will assume that the ratio $(T-D) / m$ is a perturbation, which will be denoted $a(t)$ :

$$
a(t) \stackrel{\text { def }}{=} \frac{T-D}{m} \Rightarrow \dot{V}=a(t)-g \gamma
$$

In terms of Laplace transform, the preceding equation reads as follows, where $s$ denotes the Laplace variable:

$$
s V(s)=a(s)-g \gamma(s)
$$

We then get the following block diagram for the aircraft seen as an open loop system:

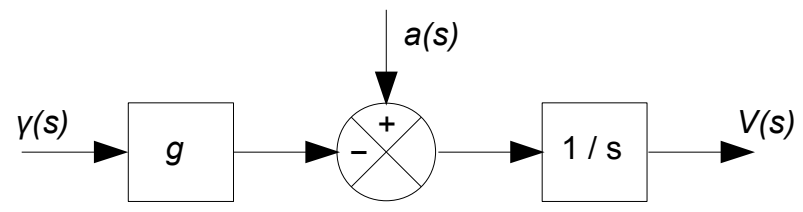

Fig. 4: block diagram of the aircraft model

One way to design a speed control law which is driven by the commanded flight path angle $\gamma_{c}$ is to use a controller $C(s)$. This controller will drive the inner loop control systems of the aircraft which compute the elevator deflection; this will be modeled in the following by a simple first order system with time constant $\tau$.

The controller $C(s)$ has been chosen to be a proportional and derivative (PI) controller. This kind of controller is widely used in industrial control systems and uses an integrator to provide zero steady-state error ${ }^{[1]}$. The parameters $K$ and $T_{i}$ are degree of freedom which have to be set:

$$
C(s)=-K\left(1+\frac{1}{s T_{i}}\right)
$$

The block diagram of the closed loop system is the following: the controller $C(s)$ calculates the error between the actual airspeed $V$ and the desired value $V_{c}$ and minimizes the error by adjusting the control input $\gamma_{c}$ : 


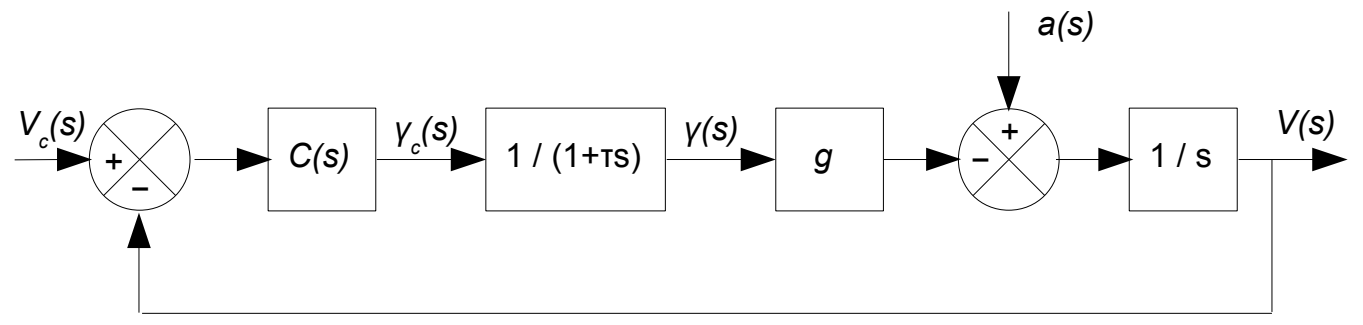

Fig. 5: block diagram of the closed loop system

Finally, an anti-windup structure ${ }^{[12]}$ has been added to prevent large transients due to the integral action which may occur when the commanded flight path angle $\gamma_{c}$ is saturated. This leads to the following block diagram, where the systems modeled through the different blocks are also highlighted:

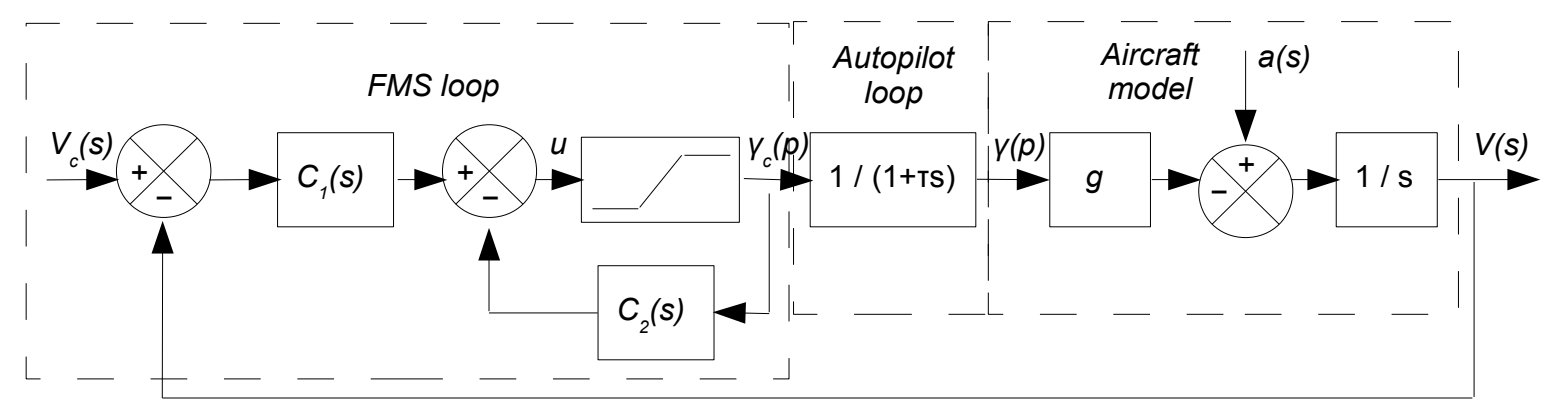

Fig. 6: block diagram of the closed loop system with an anti-windup structure

Controllers $C_{1}(s)$ and $C_{2}(s)$ have the following transfer function:

$$
\left\{\begin{array}{c}
C_{1}(p)=-K \frac{1+T_{i} p}{p-\lambda_{1}} \\
C_{2}(p)=\frac{T_{i} p}{p-\lambda_{1}}-1
\end{array}\right.
$$

The following values have been used:

$$
\left\{\begin{array}{c}
K=0.25 \\
\tau=0.3 \text { sec } \\
T_{i}=6 \quad \mathrm{sec} \\
\lambda_{1}=-0.179
\end{array}\right.
$$

Using BADA to compute idle thrust and drag of an Airbus A 320 during descent, the following figure illustrates how the controller works to change the IAS from $250 \mathrm{kts}$ to $200 \mathrm{kts}$ below 4000 feet: basically the controller stops the descent of the aircraft to allow the airspeed to decrease; then, once $220 \mathrm{kts}$ IAS is achieved, the descent continues. 


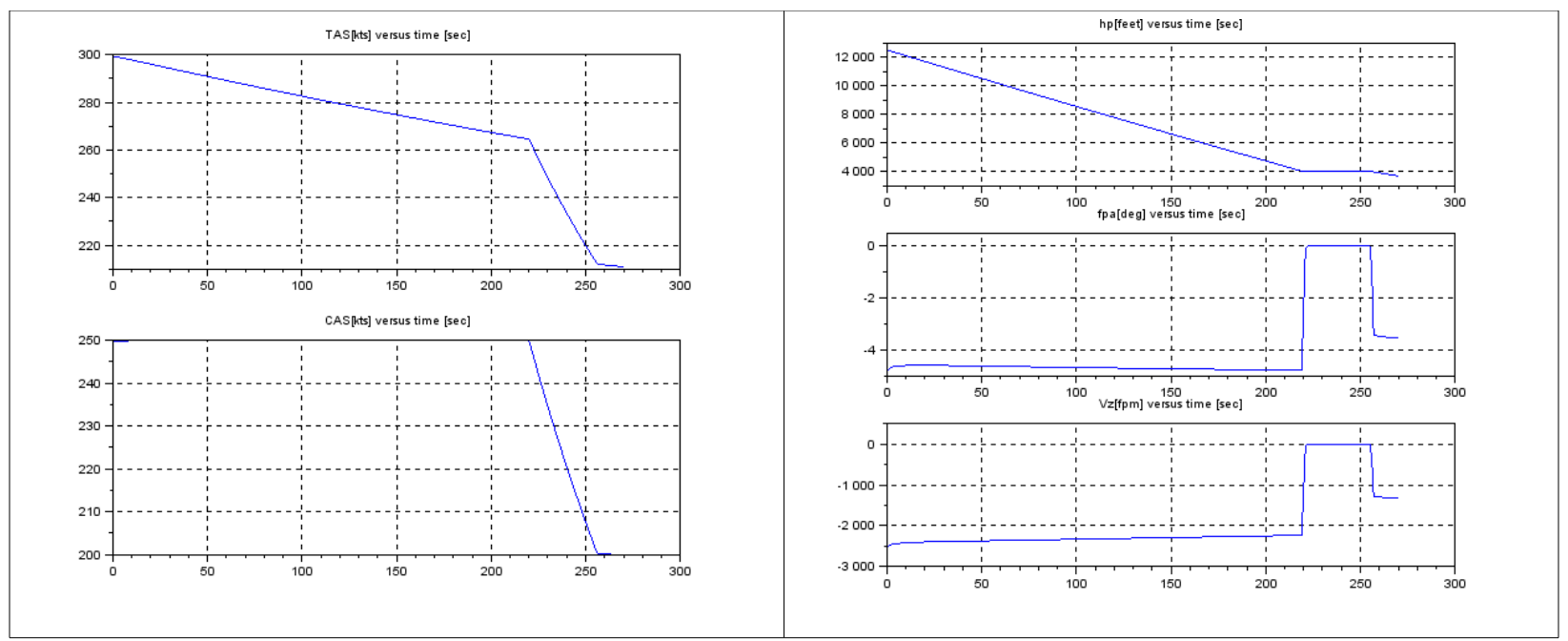

Fig. 7: block diagram of the closed loop system with an anti-windup structure

It is worth noticing that the commanded flight path angle $\gamma_{c}$ is limited between 0 and -5 degrees.

The next section is dedicated to the computation of the reference airspeed which will be used as the commanded airspeed $V_{c}$ to feed the controller (see Fig. 6).

\section{Reference Airspeed Computation}

The purpose of this section is to propose a framework to compute a reference true airspeed (TAS) such that the aircraft will overfly a specified meter at a specific time and speed. This means that we are looking for a reference airspeed $V_{r}(t)$ such that at given time $T$ the aircraft has flown a given distance $d_{f}$; in addition, we wish that the aircraft has an airspeed $V_{f}$ at time $T$. Assuming a constant wind denoted $W$, this can be written as the following two boundaries values problem:

$$
\text { find } V_{r}(t) \text { s.t. }\left\{\begin{array}{c}
V_{r}(0)=V_{0} \\
V_{r}(T)=V_{f}-W T \\
l(T) \stackrel{\text { def }}{=} \int_{0}^{T} V_{r}(t) d t=d_{f}
\end{array}\right.
$$

In the following, we will replace time $t$ by the dimensionless parameter $\tau$ which is defined by the ratio between actual time $t$ and the duration $T$ to overfly the given meter fix:

$$
0 \leq \tau \stackrel{\text { def }}{=} \frac{t}{T} \leq 1
$$

Following previous work ${ }^{[13]}$, we will consider the following expression for reference length $l(\tau)$, where parameters $a_{0}, a_{1}, a_{2}$ and $b$ are free parameters:

$$
l(\tau)=T\left(a_{0} \tau+\frac{a_{1}}{\sqrt{b}} \operatorname{atan}(\sqrt{b} \tau)+\frac{a_{2}}{\sqrt{b}}(\operatorname{atan}(\sqrt{b}(\tau-1))+\operatorname{atan}(\sqrt{b}))\right)
$$

Time derivation of the preceding equation leads to the expression of the reference airspeed:

$$
V_{r}(\tau)=a_{0}+\frac{a_{1}}{b \tau^{2}+1}+\frac{a_{2}}{b(\tau-1)^{2}+1}
$$

In order to set the initial value of the reference speed at the current speed of the aircraft and in order to satisfy the constraints presented in (17), parameters $a_{0}, a_{1}, a_{2}$ and $b$ shall satisfy the following relationships: 


$$
\left\{\begin{array}{c}
V_{r}(0) \stackrel{\text { def }}{=} V_{0} \Leftrightarrow a_{0}+a_{1}+\frac{a_{2}}{b+1}=V_{0} \\
l(1)=d_{f} \Leftrightarrow a_{0}+\frac{\operatorname{atan}(\sqrt{b})}{\sqrt{b}}\left(a_{1}+a_{2}\right)=\frac{d_{f}}{T} \\
V_{r}(1)=V_{f} \Leftrightarrow a_{0}+\frac{a_{1}}{b+1}+a_{2}=V_{f}
\end{array}\right.
$$

Assuming that parameter $b$ is already set, parameters $a_{0}, a_{1}$ and $a_{2}$ can easily be computed from the preceding equations.

The following figures shows the influence of parameter $b$ on the reference speed which satisfies the following problem:

$$
V_{r}(t) \text { s.t. }\left\{\begin{array}{c}
T=270 \text { sec } \\
V_{r}(0)=300 \text { knots TAS }(\text { i.e. } 250 \text { knots IAS at } 12500 \text { feet }) \\
V_{r}(T)=233 \text { knots TAS }(\text { i.e. } 220 \text { knots IAS at } 4000 \text { feet }) \\
l(T) \stackrel{\text { def }}{=} \int_{0}^{T} V_{r}(t) d t=20 \text { NM }
\end{array}\right.
$$

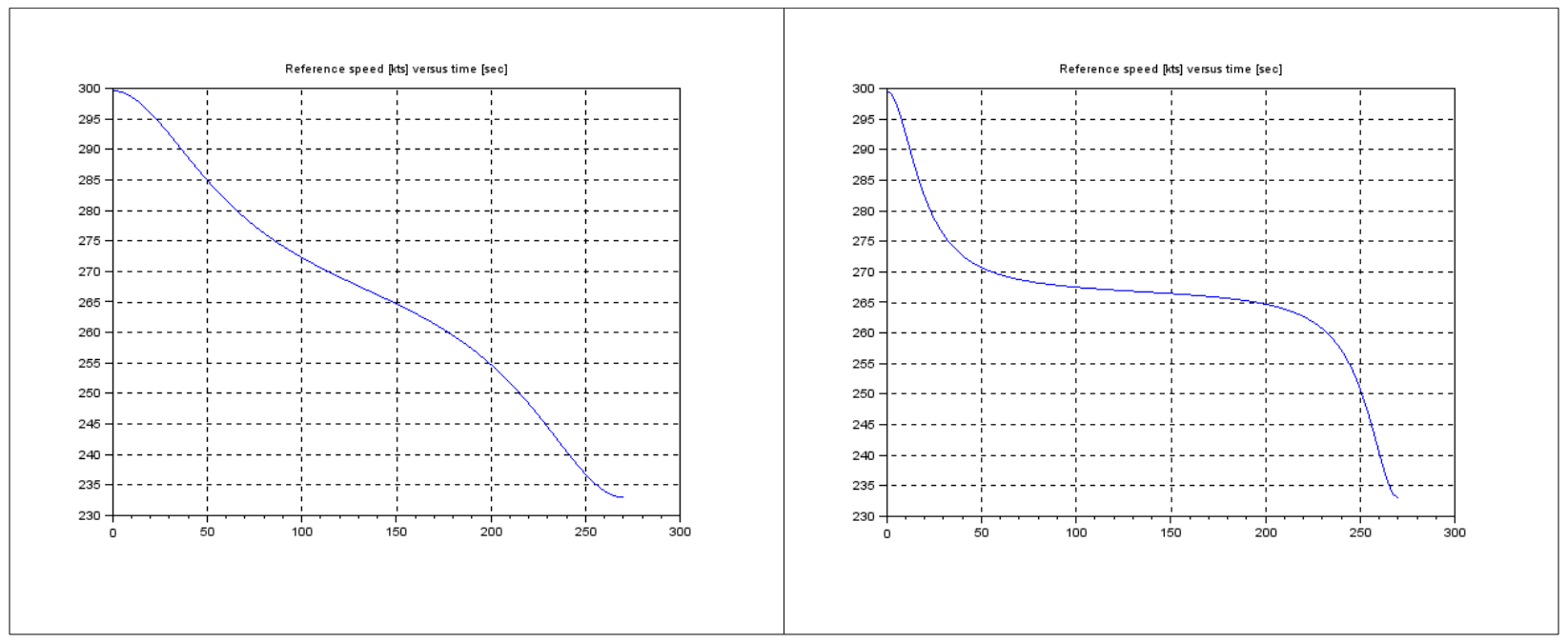

Fig. 8: influence of parameter $b$ on the reference airspeed ( $b=20$ on the left, $b=200$ on the right)

It can be seen that the greater parameter $b$ is, the higher the maximal acceleration of the reference speed is; indeed, the area $\int_{0}^{T} V_{r}(t) d t$ (that is the mean airspeed times the duration $T$ ) shall remain constant and equal to $d_{f}$. Referring to Fig. 3, we can conclude that parameter $b$ indirectly influence the value of the flight path angle, that is the value of the achieved altitude after duration $T$. From a mathematical point of view, we have to use the following two equations, where subscript $r$ indicates that we refer to the reference trajectory: the first one deals with the kinematics expression of the rate of descent whereas the second equation deals with the aircraft dynamics involving drag:

$$
\left\{\begin{array}{c}
\dot{V}_{r}=\frac{T-D\left(V_{r}\right)}{m}-g \sin \left(\gamma_{r}\right) \Rightarrow h_{r}(t)=h_{0}+\int_{0}^{t} \frac{V_{r}(\tau)}{g}\left(\frac{T-D\left(V_{r}(\tau)\right)}{m}-\dot{V}_{r}(\tau)\right) d \tau \\
\dot{h}_{r}=V_{r} \sin \left(\gamma_{r}\right)
\end{array}\right.
$$

It is clear that any change in the reference speed $V_{r}$ induces a change in the rate of descent and altitude at time $t$.

Last but not least, we also wish to set the altitude of the aircraft at time $T$; this will be done by the adjustment of the instant at which the aircraft changes from level flight to descent; The search is simply done by a dichotomic algorithm. The fine tuning of the final altitude is dedicated to remaining parameter $b$ which is adjusted to achieve the desired altitude $h_{f}$ at time $t_{f}$. 


\section{Simulation results}

In this section, some test scenarios are designed in order to illustrate the properties of the control architecture previously presented. The starting altitude is 10000 feet, the initial airspeed is 250 knots calibrated airspeed (CAS) and the distance to be flown by the aircraft before reaching the meter fix is $20 \mathrm{NM}$; in addition, the meter fix is assumed to be situated at $13 N M$ of the runway threshold. We wish that the Airbus A 320 aircraft model overfly the meter fix after $270 \mathrm{sec}$ of flight: this time is assumed to be specified by the air traffic controller through an Arrival Manager (AMAN). We also impose that the airspeed of the aircraft over the fix is 220 knots CAS and its altitude is 4000 feet so that the aircraft will be correctly prepared for landing.

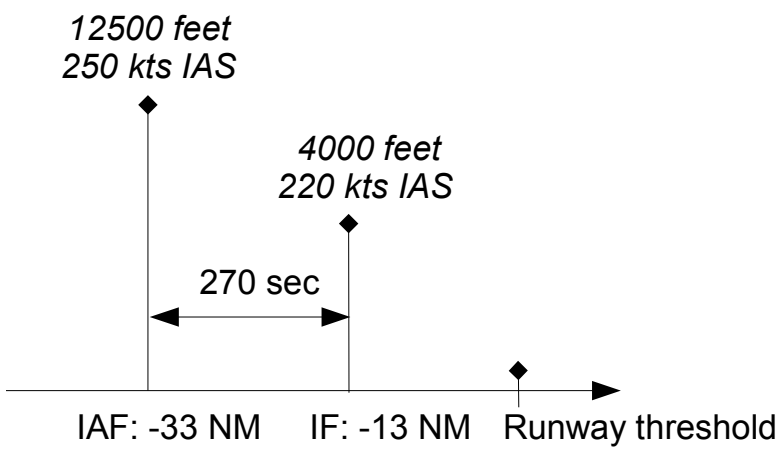

Fig. 9: Vertical profile constraints

First of all, let us analyze what happen without any reference speed: the CAS is simply set to $220 \mathrm{kts}$ at the beginning of the simulation: the following figure shows that after $270 \mathrm{sec}$ of flight, and assuming no wind, the altitude of the aircraft is 3048 feet (about 1000 feet under the targeted altitude of 4000 feet) and the distance flown is 18.3 NM, that is that the aircraft is about $2 N M$ before the IF (20 NM have to be flown to overfly the IF): consequently, without reference speed the aircraft is too far from the IF and too low:
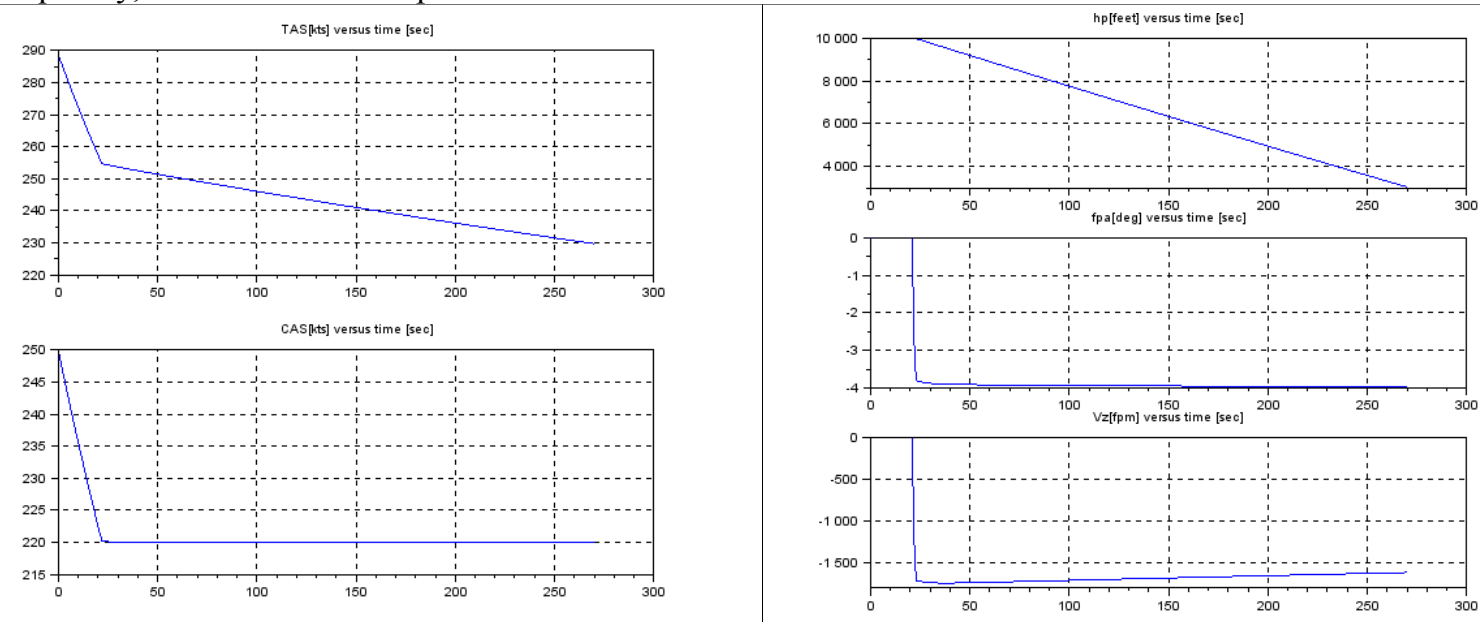

Fig. 10: Simulation results without reference speed - no wind

In order to comply with the constraints summarized in Fig. 9, we use a reference speed computed as described in the previous section. The following figures show the reference airspeed which has been computed as well as the actual airspeed (CAS and TAS, i.e. true airspeed) and the vertical profile of the descent when no wind is assumed; here parameter $b$ has been set to 20. It is worth noticing that the aircraft is maintained at level flight during $67 \mathrm{sec}$ before starting the descent; as explained before, the instant of the descent is part of the control scheme and is computed through a dichotomic manner: 

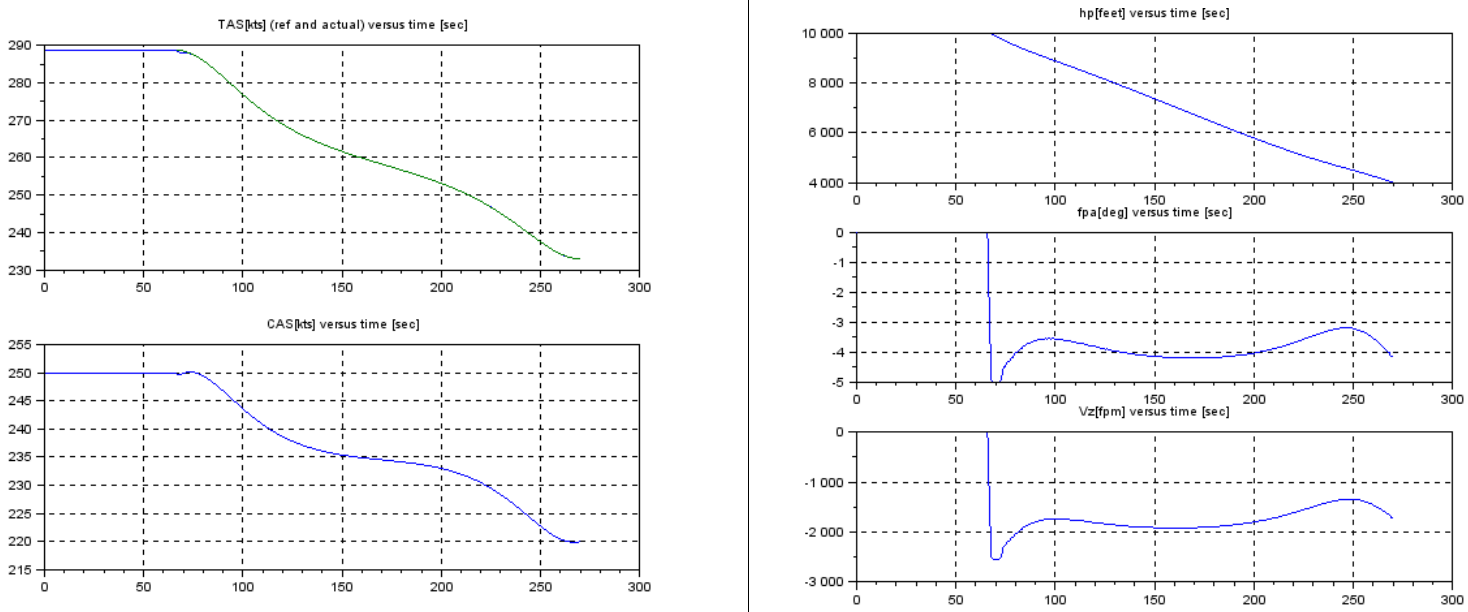

Fig. 11: Simulation results with $b=20$ and no wind

In the following simulation, the parameter $b$ has been changed to 100 ; thus the instant at which the aircraft start to descent has slightly moved to $66 \mathrm{sec}$; as expected, the maximum acceleration of the reference speed (TAS and CAS) is higher compared to the case where $b=20$; in addition, the value of the indicated airspeed (CAS) slightly increases and then decreases during the descent, which is not satisfactory from an operational point of view. Indeed, the proposed approach focuses on the shaping of the true airspeed, but the coupling between aircraft altitude during descent and computed true airspeed may result in a decreasing and then increasing calibrated airspeed (CAS) during descent which may be disturbing for the aircrew.

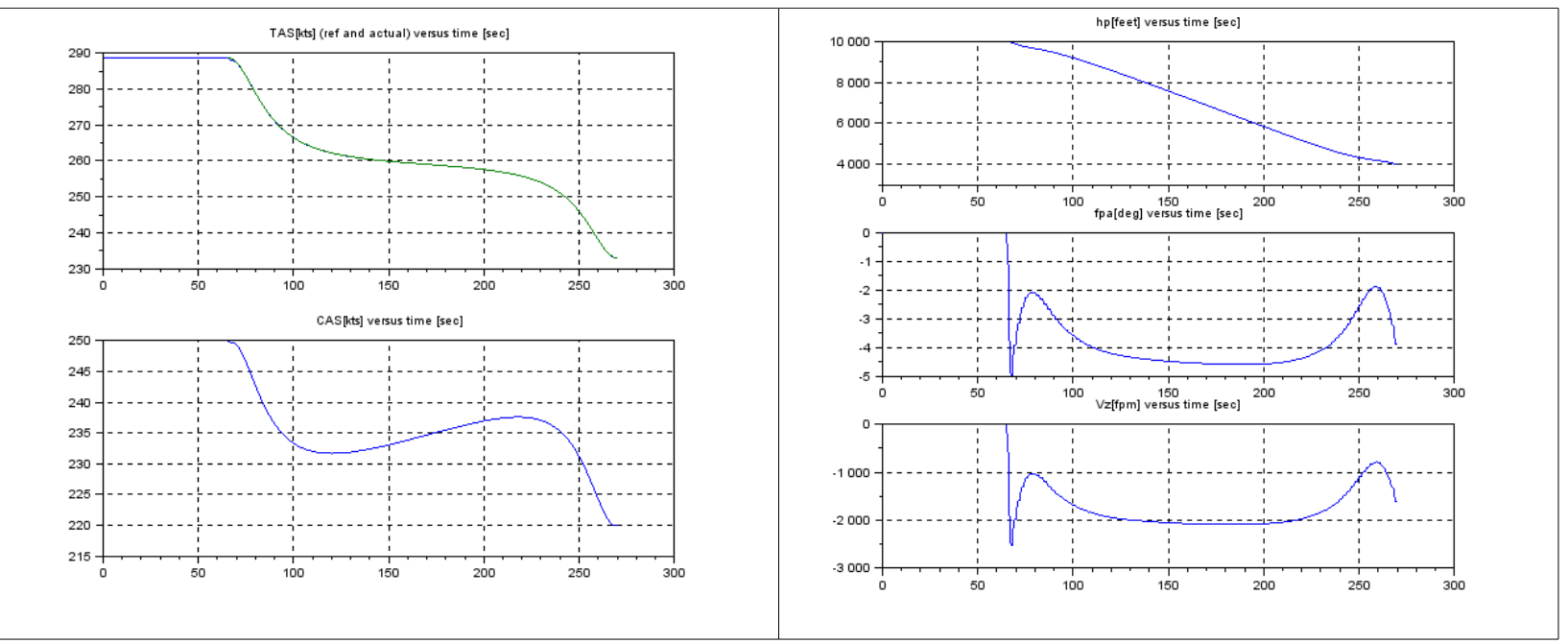

Fig. 12: Simulation results with $b=100$ and no wind

The last simulation presents the results with a front wind of 20 knots while maintaining parameter $b=100$. We can see on the following figures that the descent begins after $104 \mathrm{sec}$ of flight and that the actual airspeed does not follow exactly the reference airspeed; this comes from the saturation of the commanded flight path angle, which is not taken into account in the computation of the reference airspeed (TAS); as a consequence, while an altitude of 4000 feet is achieved over the meter fix, the constraints to fly $20 \mathrm{NM}$ in $270 \mathrm{sec}$ and to overfly the meter fix at 220 knots CAS are not met. This issue can be overcome through a periodic update on the computation of the reference airspeed but saturation of the actual airspeed produce operational constraints which shall be indicated to the aircrew. In addition, the restriction at 250 knots IAS below FL 100 is not possible due to the short duration $(270 \mathrm{sec})$ imposed for the descent. 


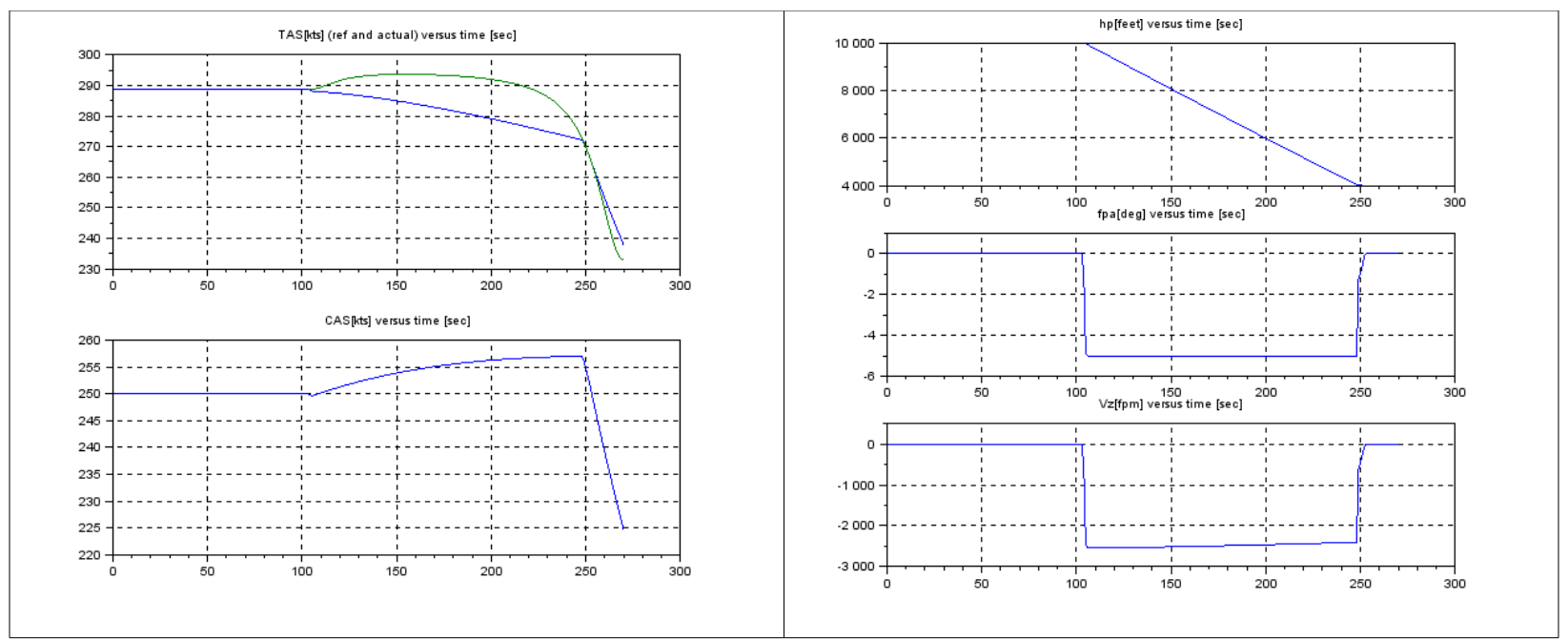

Fig. 13: Simulation results with $b=100$ and 20 knots of front wind

\section{Conclusion}

In this paper, the design of a new autopilot mode dedicated to the achievement of the overflown of a meter fix at a specified time, airspeed and altitude has been considered. This new time based autopilot system could be the airborne counterpart of the ground based arrival manager (AMAN).

This paper has considered a 2 dimensional movement, including constant wind. The setting of the reference airspeed to solve the two points boundaries problem has been considered. The proposed approach is based on the shaping of the reference airspeed, which is actually the controlled true airspeed (TAS), through the commanded flight path angle, assuming idle thrust. The coupling between aircraft descent and computed true airspeed may result in a decreasing and then increasing calibrated airspeed (CAS) during descent which may be disturbing for the aircrew.

Simulation results illustrates the efficiency of the proposed design with respect to the time and speed constraints; nevertheless, the available degree of freedom in the computation of the reference airspeed (parameter $b$ ) does allow a very little flexibility to achieve the desired final altitude and could only be used for fine tuning. The constraint on the final altitude has been achieved through the adjustment of the duration of the level flight before starting the descent, and thus by moving the thrust from idle to the necessary thrust to maintain level flight. It could also be done through aircraft configuration (flaps, landing gear).

The proposed approach can be extended to the case where constraints over multiple fixes are imposed. Indeed, the way to compute the reference airspeed takes explicitly into account the initial and final values for position and airspeed of the aircraft and can thus be used to accommodate the reference airspeed to each segment of flight.

In addition, saturation of the actual airspeed may produce operational constraints which shall be indicated to the aircrew. The robustness of the proposed design with respect to unexpected wind or saturation of the commanded flight path angle can be achieved by the periodic update of the computation of reference airspeed. This will be tested when a 3 dimensional movement will be considered with gradient of wind; in addition, during an aircraft turn, relative wind will change and an update of the computed reference speed will be needed.

Future developments are expected to realize the potential performance of CDO without compromising the optimal Airport Arrival Rate (AAR). Standardization of procedures is also very important for flight safety and need to be designed and presented in an unambiguous manner. 


\section{References}

1 ICAO, Continuous Descent Operations (CDO) Manual, Doc 9931, 2010

2 Zhou Jun, Wang Ning, Time Constrained Continuous Descent Approach, ENAC internal report, January 2013

3 Heathrow Airport Flight Evaluation Data Report Q1 2011-

http://www.heathrowairport.com/static/Heathrow_Noise/Downloads/PDF/FlightEvaluationDataReport-Q12011_LHR.pdf

4 Yi Cao, Tatsuya Kotegawa, Joseph Post, Evaluation of Continuous Descent Approach as a Standard Terminal Airspace Operation, Ninth USA/Europe Air Traffic Management Research and Development Seminar, June 14-17 2011, Berlin, Germany

5 Mueller K. T., Bortins R., Schleicher D. R. and Sweet D., Effect of Uncertainty on En Route Descent Advisor (EDA) Predictions, AIAA 4th Aviation Technology, Integration and Operations (ATIO) Forum, 20 - 22 September 2004, Chicago, Illinois

6 Sai Vaddi, Gregory D.Sweriduk, Monish Tandale, 4D Green Trajectory Design for Terminal Area Operations Using Nonlinear Optimization Techniques, AIAA, Guidance, Navigation, and Control Conference, 13-16 August 2012, Minneapolis, Minnesota

7 Boursier L., Favennec B., Hoffman E., Trzmiel A., Vergne F., Zeghal K., Merging Arrival Flows Without Heading Instructions, 7th USA/Europe Air Traffic Management R\&D Seminar, Spain, July 2007

8 Park S. G. and Clarke J.P., Vertical Trajectory Optimization for Continuous Descent Arrival Procedure, AIAA Guidance, Navigation, and Control Conference, Minneapolis (Minnesota - USA), Paper 2012-4757, 2012

9 Miquel T., 4D Green Approach Trajectory: Illustrative Assessment On Toulouse Approach, AIAA, Guidance, Navigation, and Control Conference, 2010

10 EEC Technical/Scientific Report No. 2009-003, User Manual For The Base Of Aircraft Data (BADA) revision 3.7, Eurocontrol Experimental Centre

11 Karl Johan Aström, Richard M. Murray, Feedback Systems: An Introduction for Scientists and Engineers, Princeton Univeristy Press, 2009

12 Middleton, R. H. (1996), The Control Handbook, chapter 20.1 Dealing with Actuator Saturation, pp. 377381. CRC Press, Boca Raton, Florida.

13 Miquel T., A Flatness Based Speed Control Approach For Merge Behind Operations, AIAA Guidance, Navigation, and Control Conference, 13 - 16 August 2012, Minneapolis, Minnesota

14 Nikoleris T., Chatterji G., Coppenbarger R., Comparison of Fuel Consumption of Descent Trajectories under Arrival Metering, AIAA Guidance, Navigation, and Control Conference, 13 - 16 August 2012, Minneapolis, Minnesota 